\title{
Macrophage Functions Are Regulated by the Substratum of Murine Decidual Stromal Cells
}

\author{
Raymond W. Redline, ${ }^{\star *}$ Dianne B. McKay, ${ }^{\ddagger}$ Miguel A. Vazquez, \\ With the technical assistance of Colleen $M$. Shea and Sherri R. Schmalzried \\ Departments of Pathology* and Medicine (Renal Division), ${ }^{\ddagger}$ Brigham and Women's Hospital, Boston, Massachusetts 02115; Division \\ of Nephrology, University of Texas Southwestern Medical School, Dallas, Texas 75235; and Department of Pathology," \\ Tufts University Schools of Medicine and Veterinary Medicine, Boston, Massachusetts 02111
}

\begin{abstract}
Because of their paternal antigens, the fetus and placenta may be considered an allograft in the maternal host. Local properties of the maternal-fetal interface, the placenta and decidua basalis, are important in preventing maternal immunologic rejection of the fetoplacental allograft. However, the exact nature of these local properties remains a fundamental unsolved problem in immunology. We now report that three macrophage functions were inhibited by the substratum formed by monolayers of decidual stromal cells via a novel pathway. Solidphase inhibitors blocked macrophage adhesion, spreading, and lysis of tumor necrosis factor- $\alpha$-resistant P815 mastocytoma tumor cells. Inhibition was not solely attributable to an inability of macrophages to adhere to decidual substratum because there were differences in macrophage functions on this surface versus polyhema where no adherence occurred. Because macrophages play a central role in cell-mediated immunity, including allograft rejection, inhibiting their function in the decidua basalis may help prevent maternal antifetal responses. (J. Clin. Invest. 1990. 85:1951-1958.) allograft • decidua basalis $\bullet$ macrophages $\bullet$ placenta $\bullet$ rejection
\end{abstract}

\section{Introduction}

The decidua basalis is where the placenta is anchored to the transformed endometrial stroma of the maternal uterus. We have recently shown that this is the only uteroplacental region in mice where fetal class I MHC-bearing cells are in direct contact with maternal cells (1). Understanding this tissue is a challenge to transplant biology because it is one of the few situations where semiallogeneic cells coexist without immunologic rejection. In previous experiments we probed the ability of the immune system to function in the murine decidua basalis by introducing Listeria monocytogenes (2), a powerful stimulus for macrophage and $T$ cell responses. In contrast to the vigorous effective anti-Listeria response in the maternal spleen and liver, macrophages and $\mathrm{T}$ cells failed to respond in the decidua basalis (3). This suggested the presence of local immunoregulators in the decidua basalis which may ordinarily prevent maternal antifetal immune responses.

Address reprint requests to Dr. Lu, now at the Division of Nephrology, Department of Medicine, University of Texas Southwestern Medical Center, 5323 Harry Hines Blvd, Dallas, TX 75235-9030.

Received for publication 12 June 1989 and in revised form $30 \mathrm{De}$ cember 1990.

J. Clin. Invest.

(c) The American Society for Clinical Investigation, Inc.

0021-9738/90/06/1951/08 $\$ 2.00$

Volume 85, June 1990, 1951-1958
Subsequent studies using deciduoma of pseudopregnancy, which closely mimic the decidua of true pregnancy, showed that maternal decidual cells alone could account for the defective anti-Listeria response (4). The failure of macrophages to respond to listeria in decidual tissues is unique and cannot be explained by the absence of $\mathrm{T}$ cells. Even without functional $\mathrm{T}$ cells, mice with severe combined immune deficiency mount a macrophage response against listeria (5). We believe that these unique properties of decidua may help explain the absence of maternal antifetal immune responses and contribute to the increased susceptibility of the fetoplacental unit to infection.

We now report that macrophages in decidua may be inhibited by a novel pathway. Decidual stromal cells (DEC) ${ }^{1}$ form a "substratum" in vitro which consists of the cells themselves, their cell surfaces, and their associated extracellular matrix (6). We found that this substratum inhibited three macrophage functions: macrophage-mediated tumor lysis, adherence, and spreading. Although the substratum is well known to regulate differentiation and activation of a number of leukocyte populations (7-9), this is, to our knowledge, the first report where macrophage functions are inhibited by such solid phase signals.

\section{Methods}

DEC substrata. Deciduoma were induced as previously described (4) Oophorectomized F1 (B10.A $\times$ BALB/c ByJ) female mice were treated with subcutaneous injections of estradiol $(E)$ and progesterone $(P)$ (Sigma Chemical Co., St. Louis, MO). Mice received $100 \mathrm{ng}$ of $\mathrm{E}$ on days $1-3$, no hormones on day 4-5, $1 \mathrm{mg}$ of $P+10 \mathrm{ng}$ of $E$ on days $6-8$, and $1 \mathrm{mg}$ of $P$ on days 9-10.6 h after receiving hormones on day 8 , the ends of the uterine horns were ligated and $5-10 \mu \mathrm{l}$ of peanut oil was injected between the ligatures. Animals were killed on day 11. The successful induction of deciduoma resulted in a 10-15-fold increase in average uterine weight. Decidual stroma was isolated by blunt dissection into iced $\mathrm{Ca}^{++}-, \mathrm{Mg}^{++}$-free Hanks' balanced salt solution and diced into 1-2-mm fragments with sterile razor blades. Fragments were digested with dispase $(2 \mathrm{mg} / \mathrm{ml}$ ) (Boehringer Mannheim Biochemicals, Indianapolis, IN) and DNAase ( $1 \mathrm{mg} / \mathrm{ml}$ ) (no. D0866, Sigma Chemical Co.) for $1 \mathrm{~h}$ at $37^{\circ} \mathrm{C}$, then triturated through pasteur pipettes with decreasing graded apertures. The dispersed DEC cells were filtered through Nytex, washed, and harvested from the 1.028/1.080 interface of a discontinuous Percoll gradient after centrifugation at $600 \mathrm{~g}$ for 30 min. DEC were then plated at 9,000 cells $/ \mathrm{mm}^{2}$ in Ham's F12/DME (1:1) supplemented with $20 \%$ FCS, antibiotics, and L-glutamine in 96-well plates (no. 3598, Costar Data Packaging, Cambridge, MA)

1. Abbreviations used in this paper: $\mathrm{BP}$, bacteriologic plastic; $\mathrm{DEC}$, decidual stromal cells; E, estradiol; FB, dermal fibroblasts; GM-CSF, granulocyte/macrophage colony-stimulating factor; $\mathrm{P}$, progesterone; PEC, peritoneal exudate cells; rIFN- $\gamma$, recombinant interferon- $\gamma$; TCP, tissue culture-treated plastic; TNF, tumor necrosis factor- $\alpha$. 
precoated with fibronectin $(0.02 \mathrm{mg} / \mathrm{ml}$ for $3 \mathrm{~h}$, no. F4759, Sigma Chemical Co.). Nonadherent cells were washed free after $3 \mathrm{~h}$. Substrata were used between 1 and $4 \mathrm{~d}$ after dispersal. During this time period there were no differences in the macrophage-inhibitory activity of the DEC substrata.

Characterization of DEC substrata $(1,3,4)$. DEC were cultured as described above on fibronectin-coated glass coverslips. Laminin was detected after fixation in acetone $\left(4^{\circ} \mathrm{C}, 10 \mathrm{~min}\right)$ and then using a polyclonal rabbit anti-laminin antiserum (Collaborative Research, Cambridge, MA) followed by biotinylated goat anti-rabbit IgG and peroxidase-streptavidin (Kirkegaard \& Perry Laboratories, Inc., Gaithersburg, MD). Rat hybridoma cells specific for the following antigens were obtained from the American Type Culture Collection (Rockville, MD), grown according to recommended instructions, and used as monoclonal antibody (MAb) containing tissue culture supernatants: leukocyte common antigen (MAb M1/89), Thy 1.2 (MAb 30H12), CD4 (MAb GK1.5), CD8 (MAb 2.3.4), Mac 1 (MAb M1/70), B220 (MAb T1B 164), and MAb F4/80. Primary rat MAbs were applied after fixation of decidual substrata with $2 \%$ paraformaldehyde $\left(4^{\circ} \mathrm{C}, 10 \mathrm{~min}\right)$. Biotinylated goat anti-rat IgG and peroxidase-streptavidin (Kirkegaard \& Perry Laboratories, Inc.) were applied sequentially after primary antibody. In all cases positive controls documented the efficacy of the antibodies used. Negative controls included cells known to lack antigen and isotope-matched irrelevant primary antibodies tested on DEC substrata. Peroxidase activity was detected using diaminobenzidine $\left(2 \mathrm{mg} / \mathrm{ml}\right.$, Sigma Chemical Co.), $1 \% \mathrm{H}_{2} \mathrm{O}_{2}, 0.3 \%$ sodium azide. Coverslips were counterstained with Gill-2 hematoxylin. Staining with the periodic acid-Schiff reagent was performed using standard techniques.

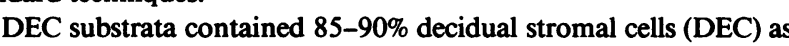
defined by the presence of immunoreactive cytoplasmic laminin (10) and typical ultrastructural criteria (11). Contaminating cells were all positive for leukocytic common antigen, F4.80, and Mac-1 identifying them as monocyte/macrophages. T and B lymphocytes and previously described decidual granulated metrial gland cells (see Bell [12] for review) were not detectable after staining for Thy 1.2, CD4, CD8, B220, and periodic acid-Schiff-positive granules.

Control substrata and other surfaces. Dermal fibroblast lines were established from F1 (BALB/c ByJ $\times$ B10.A) mice by explant culture of depilated skin from the interscapular region (13). Lines were maintained with twice weekly changes of Ham's F12/DME supplemented with $10 \%$ FCS, L-glutamine, and antibiotics. Cells were passaged every week after dispersing with 1 mM EDTA. The NIH 3T3-transformed fibroblast line was a gift from Dr. Barbara Ballerman, Brigham and Women's Hospital and was maintained in a similar fashion. MDCK epithelial cells (high electrical resistance line; 14, 15) were originally from the American Type Tissue Culture Collection. These cells were maintained in DME ( $1 \mathrm{~g} /$ liter glucose) supplemented with $5 \%$ fetal calf serum, and passaged every week after dispersing with 1 mM EDTA and $0.05 \%$ trypsin. Other surfaces included tissue-culture treated plastic (TCP) from Costar Data Packaging (no. 3596), bacteriologic plastic (BP) from Linbro (no. 76-232-05, Flow Laboratories, Inc., McLean, VA), and plastic coated with either poly-hema (Hydron, Interferon Science Inc., New Brunswick, NJ) or laminin (Collaborative Research Inc., Bedford, MA). The poly-hema surface was prepared according to the manufacturer's instructions and preincubating with $\mathrm{HBSS}$ at $37^{\circ} \mathrm{C}$ for $2 \mathrm{~h}$ before use. Laminin-coated wells were incubated overnight with a $20 \mu \mathrm{g} / \mathrm{ml}$ solution of laminin, according to the method of Mercurio and Shaw (16).

Macrophages and macrophage activating factors. Macrophageenriched peritoneal exudate cells (BALB/c ByJ $\times$ B10.A) were obtained by lavage $4 \mathrm{~d}$ after an intraperitoneal (i.p.) injection of $10 \%$ proteose peptone. Peritoneal macrophages stimulated in vivo were obtained from Listeria-immune mice. These mice were immunized with $2 \times 10^{5}$ Listeria s.c.; 2 mo later they received $2 \times 10^{5}$ Listeria i.p.; $3 \mathrm{~d}$ later they received $10 \%$ proteose peptone i.p.; and $4 \mathrm{~d}$ after this, the peritoneal exudate was harvested. RAW 264.7 is an Abelson murine leukemia virus-transformed macrophage cell line which expresses many macrophage activities including lysozyme secretion, phagocytosis, and the killing of P815 tumor targets $(17,18)$. Peritoneal and RAW 264.7 macrophages were stimulated in vitro by incubation with varying doses of macrophage activation factor (either supernatant from antigen-stimulated, D3.1 T-cells or recombinant rat interferon-gamma [IFN- $\gamma$, Amgen, Thousand Oaks, CA]), plus LPS (Escherichia coli endotoxin, no. L3254, Sigma Chemical Co.). D3.1 is a CD4 ${ }^{+}$Listeriaspecific, $\mathrm{T}$ cell clone derived in our laboratory which has been shown to produce bioactive IFN- $\gamma(19)$.

Tumor killing assay. Details of the tumor killing assay have previously been reported (20). P815 mastocytoma cells, passaged in vitro, were labeled by a 10 -min incubation with $25 \mu / \mathrm{Ci}$ of [ ${ }^{111}$ In] ]oxine (Amersham Corp., Arlington Heights, IL). After overnight culture, media was removed from the various substrata using a 25-gauge needle. $3 \times 10^{5}$ peritoneal cells and $4 \times 10^{4}{ }^{111} \mathrm{In}$-labeled P815 were then added in the presence of varying doses of IFN- $\gamma$ and LPS. Triplicate wells were sampled at 20-22 h. For RAW 264.7 cells, $3.75 \times 10^{4}$ macrophages were cultured on the substrata in the presence of stimulatory factors as above. $2 \times 10^{4} \mathrm{P} 815$ cells were added at $8 \mathrm{~h}$ and triplicate wells were sampled $16 \mathrm{~h}$ later. Percent specific release (tumoricidal index, $T I)$ was calculated from the formula $T I=100 \times(X C-S R / T C$ $S R$ ), where $X C$ is experimental counts, $S R$ is spontaneous release, and $T C$ is total counts added.

Transfer experiments were performed as follows. RAW 264.7 were cultured with stimulatory factors for $8 \mathrm{~h}$ in the 16-mm wells of BP cluster plates with or without DEC substrata, dispersed by repeated aspirations with a pasteur pipette, washed, counted, and placed on a second surface (BP, DEC substrata, or poly-hema) in 6.4-mm wells.

${ }^{111}$ In-labeled P815 were added and the ${ }^{111}$ In release measured $16 \mathrm{~h}$ later. Control experiments to exclude carryover were performed by decreasing the stimulation period from $8 \mathrm{~h}$ to $30 \mathrm{~min}$.

Transwell experiments. DEC substrata were established either above or below the polycarbonate membranes of Costar Transwells (no. 3415). These plastic inserts fit within 16-mm tissue culture wells and are separated from the bottom of the well by $1 \mathrm{~mm}$. They have a pore size of $0.6 \mu \mathrm{m}$. Tumor killing assays were performed as described above by placing RAW 264.7 macrophages, stimulatory signals, and labeled P815 cells above the Transwell membrane. After $16 \mathrm{~h}$ aliquots were removed from both above and below the Transwell. Tumoricidal indexes were calculated for RAW 264.7 macrophages in direct contact with DEC substrata and those separated from the substrata by the Transwell membrane.

Adherence assay. This assay was adapted from McClay et al. (21) and Lu et al. (20). DEC or FB established substrata in 96-well polyvinyl chloride plates (Dynatech Laboratories, Inc., Alexandria, VA) previously sterilized with $70 \%$ ethanol. Poly-hema-coated plates were also used in one experiment. RAW 264.7 macrophages were labeled with ['11'In]oxine as described above for P815 and dispensed in quadruplicate at $10^{5}$ cell-per well. After a $2-3-\mathrm{h}$ incubation at $37^{\circ} \mathrm{C}$ the wells were filled with warm media, sealed with an adhesive plastic cover, inverted, and centrifuged at varying speeds. In some cases sealed plates were simply inverted $(1 \mathrm{~g})$. After air-drying, individual wells were cut free and total bound counts were quantitated. Results were expressed as the percentage of total counts retained on each surface.

Statistical analysis. Significance to the $P=0.05$ level was ascertained using $95 \%$ confidence intervals as calculated by $1.96 \times$ standard error of the mean.

\section{Results}

Macrophages did not lyse P815 mastocytoma cells on DEC substratum. We found that macrophages did not lyse TNF-resistant (22) P815 upon substrata formed by DEC in vitro. Such lysis requires that macrophages be primed by lymphokines, such as IFN- $\gamma$, IL-4, or granulocyte/macrophage colony-stimulating factor (GM-CSF), and triggered by a second signal such as LPS (see Adams and Hamilton [23] for review). Table IA demonstrates that peptone-elicited peritoneal exudate cells 
Table I. Macrophage-mediated Tumor Lysis on Various Surfaces

\begin{tabular}{|c|c|c|c|}
\hline \multicolumn{4}{|l|}{ A. Peptone-elicited PEC } \\
\hline Surface & 0.03 & $\begin{array}{c}9 \% \\
0.30\end{array}$ & 3.00 \\
\hline TCP & $31^{*}$ & 35 & 38 \\
\hline \multicolumn{4}{|l|}{ FB } \\
\hline Subconfluent $\left(0.1 X^{\ddagger}\right)$ & 28 & 32 & 39 \\
\hline Confluent (1.0X) & 29 & 35 & 36 \\
\hline \multicolumn{4}{|l|}{ DEC } \\
\hline Subconfluent $(0.1 \times)$ & 26 & 29 & 35 \\
\hline Confluent $(1.0 \times)$ & $7^{\S}$ & $9^{8}$ & $6^{8}$ \\
\hline
\end{tabular}

LPS $25 \mathrm{ng} / \mathrm{ml}$ each well.

SEM $<5 \%$ for each triplicate sample.

* Tumoricidal index (see Methods).

$\$ 1.0 \times=$ cells plated at the standard density (see Methods); $0.1 \times$

$=$ cells plated at $10 \%$ of standard density.

${ }^{\S} P<0.05$ vs. each other group.

B. RAW 264.7 macrophage cell line

\begin{tabular}{llll} 
& \multicolumn{3}{c}{ rIFN- $\gamma$} \\
\cline { 2 - 4 } \multicolumn{1}{c}{ Surface } & 10 & $\begin{array}{l}U / m l \\
100\end{array}$ & 1,000 \\
\hline TCP & $37^{*}$ & 34 & 46 \\
FB & & & \\
Passage no. 3 & 34 & 28 & 28 \\
Passage no. 24 & 33 & 37 & 28 \\
DEC & $13^{\ddagger}$ & $8^{\ddagger}$ & $<1^{\ddagger}$
\end{tabular}

LPS $200 \mathrm{ng} / \mathrm{ml}$ each well.

SEM $<5 \%$ for each triplicate sample.

* Tumoricidal index (see Methods).

${ }^{\ddagger} P<0.05$ for DEC vs. each other group.

(PEC), a standard source of macrophages, failed to lyse P815 tumor cells on substrata formed by confluent DEC in the presence of $25 \mathrm{ng} / \mathrm{ml} \mathrm{LPS}$ and D3.1 supernatant. The latter contains IFN- $\gamma(19)$ as well as other lymphokines. On the other hand, substrata formed by subconfluent DEC and both confluent and subconfluent dermal fibroblasts (FB) supported macrophage-mediated tumor lysis as well as the control surface, TCP. (See Methods for the cellular composition of DEC substrata.)

Although enriched for macrophages, PEC are a heterogeneous cell population. We, therefore, performed parallel experiments using the macrophage cell line RAW 264.7. Table IB demonstrates that RAW 264.7 macrophages were also unable to lyse tumor targets on DEC substrata when compared to either TCP or two different FB substrata, one tested shortly after explant (passage number 3), the other after long-term tissue culture adaptation (passage number 24). Several control experiments were performed (data not shown). (a) DEC substrata were not toxic for RAW 264.7 macrophages. RAW 264.7 macrophages cultured on DEC substrata overnight were fully active when transferred and stimulated on TCP. (b) DEC did not sequester radioactive label. Supernatants containing radioactive tumor lysis produces were not depleted of counts after $16 \mathrm{~h}$ of coincubation with DEC substrata. (c) Neither the enzymes used to isolate DEC nor the age of the FB substrata had an effect on tumor lysis. Confluent FB substrata dispersed with Dispase and DNase, replated, and tested on either day 1 or day 4 were equivalent to those dispersed with EDTA and tested on the same days. Substrata formed by $3 T 3$ fibroblasts were also fully competent in supporting tumor lysis (see Table IV, discussed below).

The ideal control for DEC substrata in Fig. 1 and Table I would have been substrata formed in vitro by endometrial stromal cells from nonpregnant murine uteruses. Unfortunately, it is not possible in hormonally intact rodents to prevent such cells from differentiating into DEC in vitro. The physical trauma of dispersing these cells from uteruses is sufficient to initiate decidualization (10). If oophorectomized animals, not treated with pregnancy hormones, are used, the endometrial stromal cells do not survive well in culture (24). In the absence of a source of murine endometrial stromal cells to study in vitro, we used fibroblasts to form control substrata in Fig. 1 and Table I and found that these did not inhibit macrophage-mediated tumor lysis. Nondecidualized endometrial stromal cells and fibroblasts are both mesenchymal cells and secrete large quantities of fibronectin and type I collagen into the extracellular matrix (10).

When endometrial stromal cells from nonpregnant uteruses differentiate into decidual stromal cells, they acquire a number of epithelial characteristics, including the generation of a basement membrane type of extracellular matrix rich in laminin (10), and the formation of tight intercellular junctions
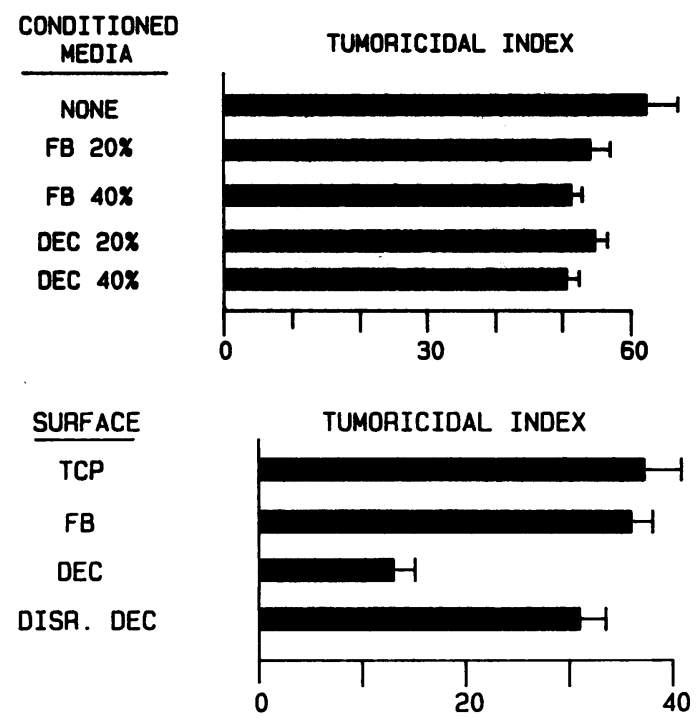

Figure 1. DEC-conditioned media and disrupted DEC substrata do not inhibit tumor lysis. (Upper panel) DEC vs. FB substrata on 16-mm wells were allowed to condition media for $18 \mathrm{~h}$. This medium was then added at the concentrations indicated to the tumor lysis assay using peptone-elicited peritoneal exudate cells as described in Table IA. Tumoricidal index was determined at $20 \mathrm{~h}$. Stimulatory signals were D3.1 supernatant (3\%) plus LPS $(25 \mathrm{ng} / \mathrm{ml}) . P>0.05$ for DEC vs. FB ( $20 \%$ and $40 \%$ ). (Lower panel) The ability of peritoneal exudate cells in the presence of $1 \%$ D3.1 supernatant and 25 $\mathrm{ng} / \mathrm{ml}$ LPS to lyse P815 (tumoricidal index) was compared in control wells (TCP or FB) vs. wells containing either intact DEC substratum or wells containing DEC substratum disrupted (DISR.) with an Eppendorf pipette to expose the underlying tissue culture plastic. $P$ $<0.05$ for DEC vs. each other group. 
(12). We therefore asked if the inability of macrophages to lyse P815 upon DEC substrata was a general property of all epithelial substrata. That this was not the case is demonstrated in Table II. Macrophage-mediated tumor lysis was as efficient upon substrata formed by the epithelial cell line MDCK (14, 15) as on TCP.

Laminin is a major component of DEC substrata (10) as well as all other basement membrane extracellular matrices (25). We examined macrophage-mediated tumor lysis on laminin-coated surfaces to determine if laminin in the DEC substratum was responsible for inhibiting macrophage-mediated tumor lysis. Table III shows that lysis of P815 was as efficient on laminin surfaces as on TCP. This concurs with published data that laminin-coated surfaces facilitate macrophage-mediated lysis of some tumors (26), and indicates that laminin, by itself, did not account for the inability of macrophages to lyse P815 on DEC substrata.

Inhibition of macrophage-mediated tumor lysis by DEC substrata required contact between macrophage and substratum. Three lines of evidence indicate that DEC did not secrete stable soluble inhibitors. Coculture experiments shown in Table IV indicate that inhibition cannot be transmitted across a polycarbonate filter (0.6- $\mu \mathrm{m}$ pore size). RAW 264.7 macrophages were stimulated by rIFN- $\gamma$ and LPS upon DEC substratum (upper portion of the table) or separated from the substratum by $1 \mathrm{~mm}$ plus the $10-\mu \mathrm{m}$ width of the filter (lower portion). DEC substrata were inhibitory only when they were in direct contact with the RAW 264.7 macrophages. Fig. 1 (top) shows that inhibition could not be transferred by conditioned media. Fig. 1 (bottom) demonstrates that confluent substrata of DEC were required to inhibit macrophage-mediated lysis. If the confluent DEC substratum was disrupted such that macrophages were able to adhere to the underlying plastic, no inhibition occurred. Taken together these experiments argue against a stable soluble mediator and suggest that intimate contact between DEC substratum and macrophages is required to prevent tumor lysis. They also indicate that DEC did not act by depleting the media of factors required for tumor lysis.

DEC substratum blocked macrophage effector function but not macrophage stimulation. The above results did not discriminate between the ability of macrophages to receive stimulatory signals and their ability to subsequently lyse tumor cells.

Table II. Macrophage-mediated Tumor Lysis Not Inhibited by Substratum Formed by MDCK Cells

\begin{tabular}{rcc}
\hline \multicolumn{3}{c}{ Tumoricidal index } \\
\cline { 2 - 3 } LPS $^{*}$ & On MDCK substratum & On TCP \\
\hline$n g l m l$ & & \\
10,000 & $60 \pm 4$ & $57 \pm 2$ \\
1,000 & $57 \pm 2$ & $58 \pm 4$ \\
200 & $61 \pm 3$ & $55 \pm 4$ \\
40 & $46 \pm 1$ & $40 \pm 5$ \\
8 & $50 \pm 6$ & $34 \pm 4$ \\
\end{tabular}

* RAW 264.7 macrophages also stimulated by $100 \mathrm{U} / \mathrm{ml}$ rIFN- $\gamma$. ‡ Substratum formed by confluent layer of MDCK cells after overnight culture.
Table III. Macrophage-mediated Tumor Lysis Not Inhibited on Laminin-coated Surfaces

\begin{tabular}{ccc}
\hline & \multicolumn{2}{c}{ Tumoricidal index } \\
\cline { 2 - 3 } LPS $^{*}$ & On TCP & $\begin{array}{c}\text { On laminin-coated } \\
\text { surface }^{*}\end{array}$ \\
\hline$n g / m l$ & & \\
2,000 & $54 \pm 1$ & $52 \pm 1$ \\
200 & $50 \pm 1$ & $47 \pm 2$ \\
20 & $46 \pm 2$ & $52 \pm 1$ \\
2 & $38 \pm 2$ & $40 \pm 1$ \\
.2 & $34 \pm 2$ & $38 \pm 1$ \\
\hline
\end{tabular}

* RAW 264.7 macrophages also stimulated by $100 \mathrm{U} / \mathrm{ml}$ rIFN- $\gamma$.

‡ See Methods.

In Fig. 2 (top), peritoneal macrophages taken from Listeriainfected mice, and thus stimulated in vivo, were assayed at various effector to target ratios on DEC vs. FB substrata in the absence of additional activating signals. Although these macrophages were fully stimulated in vivo by exposure to Listeria and IFN- $\gamma$ generated during the course of infection, their effector function on DEC substratum was inhibited.

Unlike PEC, the less adherent RAW 264.7 macrophages can be stimulated on BP or DEC substrata and then resuspended by vigorous washing. This allowed us to perform the transfer experiments shown in Fig. 2 (bottom). RAW 264.7, stimulated for $8 \mathrm{~h}$ on DEC substrata, washed, and then transferred to BP, lysed P815 as well as those stimulated on BP. On the other hand, RAW 264.7 stimulated on BP and transferred to DEC substrata did not lyse P815. Control groups in which RAW 264.7 were stimulated for suboptimal time periods (30 min) on DEC substrata and transferred to BP showed no tumor lysis, excluding physical carryover of stimulatory signals as a cause for activity after transfer (data not shown).

Table IV. Inhibition of Macrophage-mediated Tumor Lysis by DEC Substratum Requires Contact between Macrophage and Substratum

\begin{tabular}{|c|c|c|c|c|c|}
\hline \multicolumn{2}{|l|}{ Cells } & \multirow[b]{2}{*}{$\begin{array}{l}\operatorname{rIFN}-\gamma(U / m l) \ldots \\
\operatorname{LPS}(n g / m l) \ldots \ldots\end{array}$} & \multicolumn{3}{|c|}{ Tumoricidal index } \\
\hline $\begin{array}{c}\text { Above } \\
\text { Transwell }\end{array}$ & $\begin{array}{c}\text { Below } \\
\text { Transwell }\end{array}$ & & $\begin{array}{l}10 \\
40\end{array}$ & $\begin{array}{r}10 \\
200\end{array}$ & $\begin{array}{l}100 \\
200\end{array}$ \\
\hline$M+T+D E C$ & - & & $<1^{*}$ & $11^{*}$ & $11^{*}$ \\
\hline $\mathbf{M}+\mathbf{T}+\mathbf{F B}$ & - & & 35 & 44 & 39 \\
\hline$M+T+3 T 3$ & - & & 37 & 41 & 39 \\
\hline $\mathbf{M}+\mathbf{T}$ & DEC & & 27 & 27 & 25 \\
\hline $\mathbf{M}+\mathbf{T}$ & FB & & 28 & 36 & 30 \\
\hline $\mathbf{M}+\mathbf{T}$ & $3 T 3$ & & 28 & 31 & 31 \\
\hline
\end{tabular}

Substrata of decidual cells (DEC), dermal fibroblasts (FB), or 3T3 cells were established above or below polycarbonate (Transwell) filters (see Methods). RAW 264.7 macrophages (M) and P815 tumor cells $(\mathrm{T})$ were added above the Transwell in the presence of stimulatory signals. Tumoricidal index was determined $16 \mathrm{~h}$ later. All SEM $<5 \%$.

* $P<0.05$ for $\mathrm{M}+\mathrm{T}+\mathrm{DEC}$ vs. each other group. 

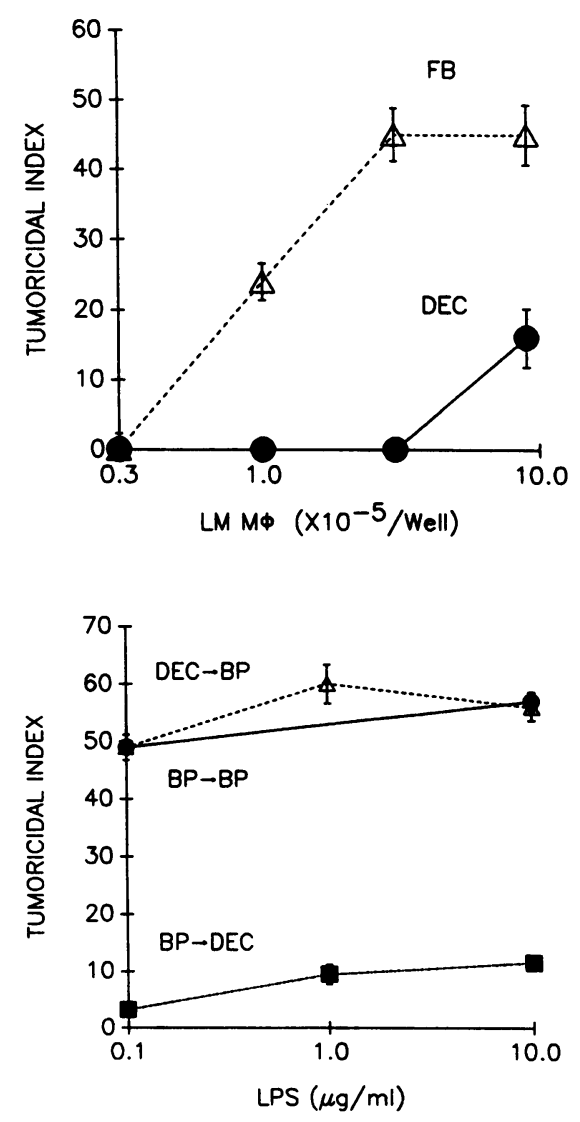

Figure 2. DEC substrata act at the effector rather than the stimulatory phase of macrophage-mediated tumor lysis. (Upper panel) Peritoneal macrophages were taken from Listeria-infected mice (see Methods). Various numbers of these in vivo stimulated macrophages (LM M0) were added to DEC vs. FB substrata in the absence of additional stimulatory signals. Tumoricidal index was determined at 16 h. $P<0.05$ for DEC vs. FB at $1.0,3.0$, and $10 \times 10^{5} \mathrm{LM}$ macrophages per well. (Lower panel) RAW 264.7 macrophages were stimulated for $8 \mathrm{~h}$ with $100 \mathrm{U}$ rIFN- $\gamma$ plus LPS in the indicated doses on BP or DEC substrata in 16-mm wells. RAW 264.7 were then dispersed, washed, and replated (as indicated by the arrows) onto either BP or DEC substrata (6.4-mm wells) in the absence of additional stimulatory signals. Tumoricidal index was determined at 16 h. $P$ $<0.05$ for BP to DEC vs. other groups at each dose of LPS.

Adherence and spreading of RAW 264.7 are inhibited on DEC substrata relative to control surfaces. Because contact was necessary to prevent macrophage-mediated tumor lysis, we investigated the effects of DEC substratum on macrophage adhesion and spreading: two early contact-mediated macrophage functions. RAW 264.7 macrophages were labeled with [ ${ }^{111}$ In] oxine and incubated on various surfaces. The wells were then sealed and centrifuged in the inverted position, thus applying a defined centrifugal force to separate macrophages from substratum. Percent adherence was determined by cutting out and counting the individual wells. Table $\mathrm{V}$ demonstrates the RAW 264.7 macrophages adhere less well to DEC substrata than to FB substrata or BP alone. Adherence to DEC substrata was $15-20 \%$ less than controls at $1 g$ and progressively decreased with increasing centrifugal force. Adherence to other surfaces was unchanged over the range of forces tested. In other experiments not shown, the media conditioned
Table V. RAW 264.7 Macrophages Adhere Less Strongly to DEC Substratum than Control Surfaces

\begin{tabular}{|c|c|c|c|c|}
\hline \multirow[b]{2}{*}{ Surface } & \multicolumn{4}{|c|}{ ['IIIn]Raw 264.7 adherence after reverse spin at } \\
\hline & 18 & $50 \mathrm{~g}$ & $200 \mathrm{~g}$ & $800 \mathrm{~g}$ \\
\hline & \multicolumn{4}{|c|}{$\%$} \\
\hline Plastic & 80 & 73 & 74 & 81 \\
\hline FB & 75 & 70 & 76 & 70 \\
\hline DEC & 61 & 48 & 43 & 35 \\
\hline
\end{tabular}

Adherent cells are expressed as the percentage of total added label remaining after centrifugation. All SEM $<5 \%$.

by DEC had no effect on adherence. A second early macrophage function, spreading, was also decreased when RAW 264.7 were plated on DEC substratum as compared to BP (Fig. 3).

Lack of adherence and spreading alone cannot account for the inhibition of tumor lysis on DEC substrata. One possible explanation for the inability of macrophages to lyse tumor cells on DEC substrata would be that macrophages must adhere to the substratum in order to function. That this was not the case was demonstrated by experiments comparing macrophage functions on DEC substrata vs. polyhema, a surface to which cells do not adhere. Table VIA shows that, as expected, RAW 264.7 macrophages did not adhere to poly-hema-coated tissue culture wells. This was in contrast to the weak, but significant, macrophage adherence to DEC substrata and the strong adherence to TCP and dermal FB substrata (Tables V and VIA). The experiment shown in Table VIB contrasts the ability of previously stimulated macrophages to kill P815 targets on poly-hema vs. DEC substrata. RAW 264.7 macrophages were stimulated on BP with varying levels of rIFN- $\gamma$ plus LPS for $8 \mathrm{~h}$, washed, and then transferred as described above to poly-hema, BP, or DEC substrata. Despite their lack of adherence, macrophage-mediated tumor lysis was as efficient on poly-hema as on BP. Macrophages transferred to DEC substrata, on the other hand, were essentially inactive.

\section{Discussion}

DEC form a substratum consisting of the cells themselves, their cell surfaces, and their extracellular matrix. We have shown that this substratum is a potent inhibitor of the effector phase of macrophage-mediated tumor lysis, a macrophage function which has been closely correlated to the ability to kill intracellular pathogens such as Listeria (23). Two other early macrophage functions, adherence and spreading, were also inhibited. This in vitro data closely parallels our previous in vivo observations that, although monocyte/macrophages translocate across endothelium, they fail to migrate from perivascular areas to sites of bacterial proliferation in the decidual tissue (3, 4). To relate the in vitro to the in vivo observations, we propose that without strong adherence macrophages lack the traction necessary for migration.

During decidualization, the fibroblast-like endometrial stromal cells differentiate into DEC and acquire many epithelial characteristics in the process (10). Because it was not possible to culture murine endometrial stromal cells without their 

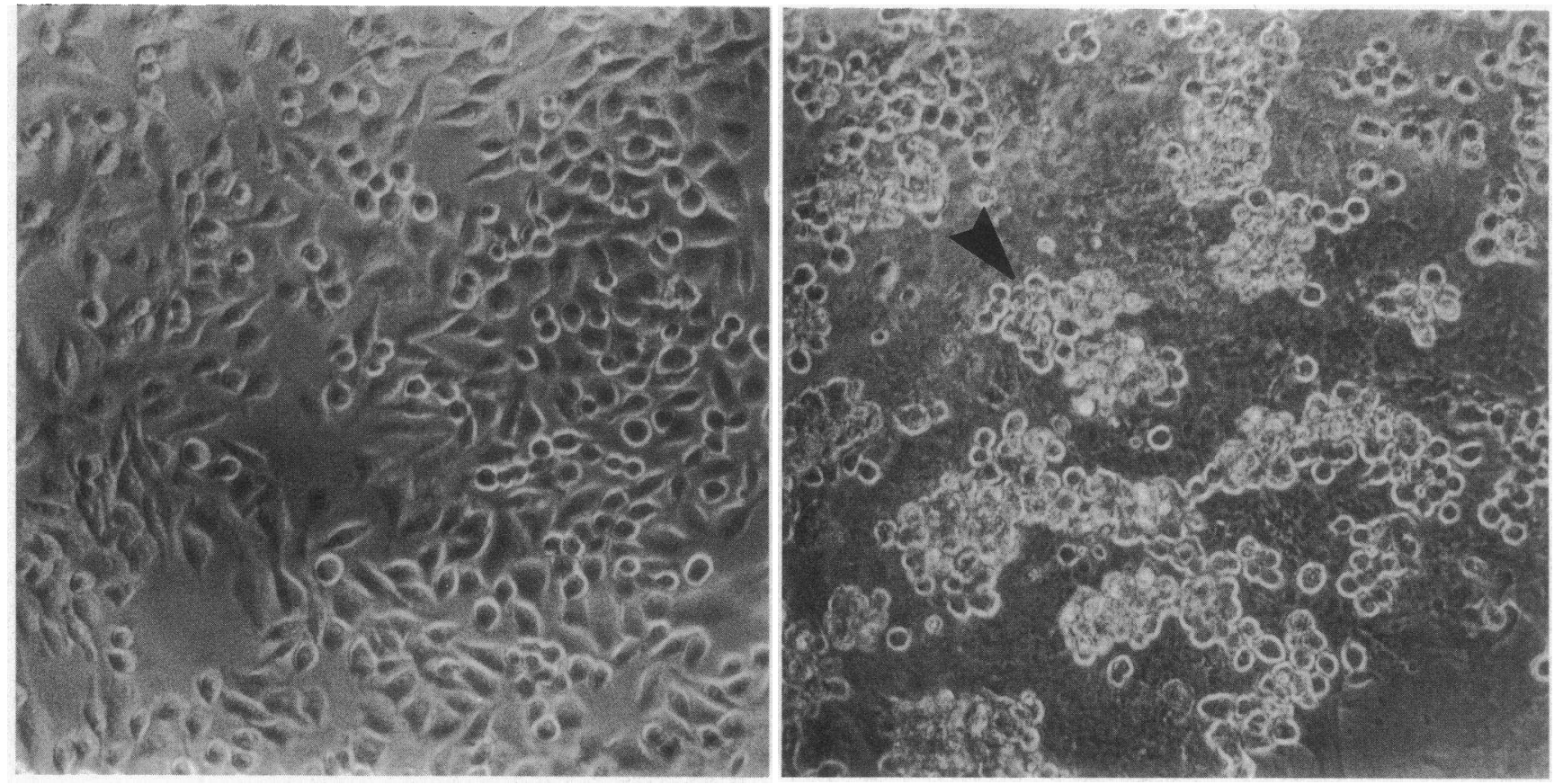

Figure 3. RAW 264.7 macrophages are less spread on the substratum formed by DEC, than on TCP. Macrophages were incubated for $16 \mathrm{~h}$ on either TCP (left) or DEC (right). Note that most macrophages have a well-spread morphology on TCP (left). In contrast, macrophages (arrow) on DEC (right) are round and clumped together. The underlying decidual substratum in the left panel is out of focus.

spontaneously differentiating into DEC (10), we used dermal fibroblast and epithelial (MDCK) cell substrata as controls. Neither inhibited macrophage-mediated tumor lysis (Tables I and II). Altogether these data suggest that just as the inability of macrophages to migrate into decidua is unique for these

Table VI. Relation of Adherence to the Ability to Support the Effector Phase of Macrophage-mediated Tumor Lysis

\begin{tabular}{lccc}
\hline \multirow{2}{*}{ A. Macrophage adherence } & \multicolumn{3}{c}{ ["1'In]Raw 264.7 adherence after reverse spin at } \\
\cline { 2 - 4 } & $200 \mathrm{~g}$ & $400 \mathrm{~g}$ & $800 \mathrm{~g}$ \\
\hline & & $\%$ & \\
Plastic & 64 & 60 & 56 \\
DEC & $27^{\S}$ & $21^{\S}$ & $19^{\S}$ \\
Poly-hema & $2^{11}$ & $1^{11}$ & $2^{11}$
\end{tabular}

B. Effector phase of macrophage-mediated tumor lysis

\begin{tabular}{lccc} 
& \multicolumn{3}{c}{ LPS } \\
\cline { 2 - 4 } Test surface* & 0.1 & $\mu g / m l$ \\
& & 1 & 10 \\
\hline BP & $49^{\ddagger}$ & 59 & 56 \\
Poly-hema & 52 & 57 & 56 \\
DEC & $3^{\S}$ & $12^{\S}$ & $12^{\S}$
\end{tabular}

rIFN- $\gamma 100 \mathrm{U} / \mathrm{ml}$, all wells.

SEM for each triplicate $<5 \%$.

* RAW 264.7 macrophages stimulated on BP and transferred to the various test surfaces (see Methods).

¥ Tumoricidal index (see Methods).

${ }^{8} P<0.05$ for DEC vs. each other group.

" $P<0.05$ for Poly-hema vs. each other group. tissues, so the inhibition of macrophage function by DEC substrata is also unique. However, these data must be interpreted cautiously because macrophages may not have access to inhibitory portions of MDCK substratum formed in vitro. Most epithelial cells, such as MDCK, grow as highly polarized monolayers with distinct basal (plastic) and lumenal (free) surfaces. Only the luminal surface is accessible to macrophages in culture. On the other hand, DEC are not polarized $(11,24)$, and the same extracellular matrix components may be distributed on all surfaces.

Laminin is a major constituent of all basement-membrane extracellular matrices formed by epithelial cells as well as DEC $(10,25)$. Macrophages adhere well to laminin surfaces only after stimulation by IFN- $\gamma$ and/or LPS $(20,27,28)$. Although laminin may contribute to the decreased macrophage adherence and spreading on DEC substrata, Table III indicates that macrophage-mediated tumor lysis occurred effectively on laminin-coated surfaces, indicating that laminin, by itself, was not responsible for the inhibitory effect of DEC substratum on the latter macrophage function.

The DEC substratum consists of the cells and their extracellular matrix. We have not yet determined which is responsible for inhibiting macrophage function. In some previous studies $(29,30)$, it has been possible to remove the cultured cells with mild maneuvers such as EDTA, urea, or trypsin, and demonstrate that the remaining extracellular matrix and associated growth factors are biologically active. However, we were unable to remove decidual cells using these maneuvers. After removing DEC using more vigorous digestion with dispase, we found that the remaining elements of the substratum were not inhibitory (data not shown). We do not believe this result is interpretable. DEC substratum is several cell layers thick (24) and contains large amounts of basement-membrane extracellular matrix. The vigorous digestion using dispase which is 
necessary to remove DEC cells would also be expected to destroy the extracellular matrix. Other experiments to determine if DEC cells or their extracellular matrix are required to inhibit macrophage activities are ongoing in our laboratory.

Table VI and Fig. 2 indicate that DEC substrata inhibited the effector phase of macrophage-mediated tumor lysis. Several explanations may be considered. One would be that the inability of macrophages to adhere and spread upon DEC substrata would interfere with their ability to form conjugates with and thus lyse the P815 targets. However, Table VIA indicates that macrophages were significantly more adherent to DEC substrata than poly-hema, but that lysis of P815 was not inhibited on the latter (Table VIB). In a similar fashion, macrophages also do not adhere well to agarose yet effector function on this surface is also not inhibited (31). These data are important in that they indicate significant differences between macrophage interactions with poly-hema and agarose, on the one hand, vs DEC substrata on the other hand. Insofar as poly-hema and agarose are "nonadherent" surfaces for macrophages, the data suggest that the inability of macrophages to lyse P815 on DEC substrata results not from the simple absence of adherence but from an inhibitory signal.

Furthermore, one must consider that macrophage activation, as assayed by tumor lysis of tumor necrosis factor (TNF)-resistant P815 (22), continues after the IFN- $\gamma$ and LPS signals have been delivered. For example, secretion of cytolytic proteases depends upon signals delivered by the tumor target $(32,33)$. These or other tumor-derived signals might be inhibited by DEC substrata.

Several direct inhibitors of macrophage function have been described including transforming growth factor- $\beta$ (TGF- $\beta$ [34]), prostaglandin $E_{2}$, p15E retroviral peptides (35), macrophage deactivating factor (36), and IFN- $\beta$ (37). Unlike the inhibitor in this report, these factors are all active-in conditioned media, and generally block stimulation as well as effector function. Prostaglandin $\mathrm{E}_{2}$ and transforming growth factor- $\beta$ have been directly evaluated in our tumor killing assay and had no suppressive activity (unpublished). It remains possible that one or more of these factors might either be concentrated by or activated upon binding to decidual substratum. Furthermore, we would like to emphasize that the inhibitor described here is distinct from previously described factors produced by decidual leukocytes (see Hunziker and Wegmann [38] for review). Inhibitors produced by decidual leukocytes block $\mathrm{T}$ cell but not macrophage functions (39), and are all transferrable in conditioned media.

Table IV indicates that contact between macrophage and some solid-phase inhibitor within the DEC substratum was necessary for inhibition. Such solid-phase, rather than freely diffusible, signals offer significant advantages in a complex organ such as the placenta. Inhibition of macrophage function can be spatially limited to the area where maternal and fetal cells are intimately intermixed while other immediately adjacent regions, which contain exclusively maternal or fetal cells, retain the capacity to support effective macrophage-mediated host responses (3). Insofar as macrophages play important antigen-presenting and effector functions in allograft rejection (40), such solid-phase mediators may help prevent maternal antifetal responses. Macrophages are a major source of proinflammatory mediators, such as IL-1, TNF, and IL-6. Excluding macrophages from the murine decidua should result in lower local amounts of these mediators.
The maternal-fetal immunologic relationship in the placenta and decidua basalis is complex. Multiple mechanisms preventing maternal antifetal responses have been suggested (see Hunziker and Wegmann [38] and Head et al. [41] for review). We propose that solid-phase inhibition of macrophage function by the decidual substratum acts in concert with these other mechanisms. The relative roles of each may depend on the species involved, the stage of pregnancy, and the anatomic location within uteroplacental tissues.

\section{Acknowledgments}

This work was supported by grants RO-1-HD-24797 and 2T32-DK07527-03 (to Dr. McKay) from the National Institutes of Health, the Hood Foundation, the Hearst Foundation, and the March of Dimes. Dr. Redline is the recipient of a National Institutes of Health Clinical Investigator Award (KO-8-HD-00864). Dr. Lu was a Fellow of the Hartford Foundation and subsequently recipient of a National Institutes of Health Research Career Development Award (KO4-HD00862). Dr. Vazquez was supported by the Kidney and Pancreatic Transplant Grant from the State of Texas.

\section{References}

1. Redline, R. W., and C. Y. Lu. 1989. Localization of fetal major histocompatibility complex (MHC) antigens and maternal leukocytes in the murine placenta: implications for understanding the regulation of maternal anti-fetal immune responses. Lab. Invest. 61:27-36.

2. Redline, R. W., and C. Y. Lu. 1988. Role of local immunosuppression in murine fetoplacental listeriosis. J. Clin. Invest. 79:12341241

3. Redline, R. W., and C. Y. Lu. 1988. Specific defects in the anti-listerial immune response in discrete regions of the murine uterus and placenta account for susceptibility to infection. J. Immunol. 140:3947-3955.

4. Redline, R. W., C. M. Shea, V. E. Papaioannou, and C. Y. Lu. 1988. Defective anti-listerial responses in deciduoma of pseudopregnant mice. Am. J. Pathol. 133:485.

5. Bancroft, G. J., M. J. Bosma, G. C. Bosma, and E. R. Unanue. 1986. Regulation of macrophage Ia expression in mice with severe combined immune deficiency: induction of Ia expression by a T-cell independent mechanism. J. Immunol. 137:4-9.

6. McClay, D. R., and C. A. Ettensohn. 1987. Cell adhesion in morphogenesis. Annu. Rev. Cell Biol. 3:319-345.

7. Nathan, C. F. 1987. Neutrophil activation on biological surfaces: massive secretion of hydrogen peroxide in response to products of macrophages and lymphocytes. J. Clin. Invest. 80:1550-1560.

8. Levi-Schaffer, F., E. T. Dayton, K. F. Austen, A. Hein, J. P. Caulfield, P. M. Gravallese, F. T. Liu, and R. L. Stevens. 1987. Mouse bone marrow-derived mast cells cocultured with fibroblasts: Morphology and stimulation-induced release of histamine leukotriene B4, leukotriene C4, and prostaglandin D. J. Immunol. 139:3431-3441.

9. Roberts, R., J. Gallagher, E. Spooncer, T. D. Allen, F. Bloomfield, and T. M. Dexter. 1988. Heparan sulfate bound growth factors: a mechanism for stromal cell mediated haematopoiesis. Nature (Lond.). 332:376-378.

10. Wewer, U. M., A. Damjanov, J. Weiss, L. A. Liotta, and I. Damjanov. 1986. Mouse endometrial stromal cells produce basement-membrane components. Differentiation. 32:49.

11. O'Shea, J. D., R. G. Kleinfeld, and H. A. Morrow. 1983. Ultrastructure of decidualization in the pseudopregnant rat. Am. J. Anat. 166:271.

12. Bell, S. C. 1985 . Comparative aspects of decidualization in rodents and human: cell types, secreted products and associated function. In Implantation of the Human Embryo. R. G. Edwards, J. M. Purdy, and P. C. Steptoe, editors. Academic Press, Ltd., London. 71-132. 
13. Geppert, T. D., and P. E. Lipsky. 1985. Antigen presentation by interferon-gamma treated endothelial cells and fibroblasts: differential ability to function as antigen-presenting cells despite comparable Ia expression. J. Immunol. 135:3750-3754.

14. Paulmichl, M., F. Friedrich, and F. Lang. 1986. Electrical properties of Madin-Darby-canine-kidney cells: effects of extracellular sodium and calcium. Pflügers Arch. Eur. J. Physiol. 407:258-263.

15. Cerijido, M., J. Ehrenfeld, I. Meza, and A. Martinez-Palomo. 1980. Structural and functional membrane polarity in cultured monolayers of MDCK cells. J. Membr. Biol. 52:147-159.

16. Mercurio, A. M., and L. M. Shaw. 1988. Macrophage interactions with laminin: PMA selectively induces the adherence and spreading of mouse macrophages on a laminin substratum. $J$. Cell. Biol. 107:1873-1880.

17. Raschke, W. C., S. Baird, P. Ralph, and I. Nokoinz. 1978. Functional macrophage cell lines transformed by Abelson leukemia virus. Cell. 15:261.

18. Gorecka-Tisera, A. M., K. W. Snowdowne, and A. B. Borle. 1986. Implications of a rise in cytosolic free calcium in the activation of RAW 264.7 macrophages for tumor cell killing. Cell. Immunol. 100:411.

19. Lu, C. Y., P. A. Lemay, and M. J. Lombardi. 1988. Inhibition of antigen-specific activation of an L3T4+ T cell line by cyclosporine with maintenance of macrophage-mediated antigen presentation. Transplantation (Baltimore). 45:187-194.

20. Lu, C. Y., M. J. Lombardi, C. M. Shea, and L. B. Dustin. 1988. High strength binding of P815 mastocytoma cells is not necessary for their lysis by macrophages which have been primed and triggered in vitro. J. Immunol. 141:1083.

21. McClay, D. R., G. M. Wessel, and R. B. Marchase. 1981. Intercellular recognition: quantitation of initial binding events. Proc. Natl. Acad. Sci. USA. 78:4976-4980.

22. Decker, T., M. L. Lohmann-Matthes, and G. E. Gifford. 1987 Cell-associated tumor necrosis factor (TNF) as a killing mechanism of activated cytotoxic macrophages. J. Immunol. 138:957-962.

23. Adams, D. O., and T. A. Hamilton. 1986. Destruction of tumor cells by mononuclear phagocytes: models for analysing effector mechanisms and regulation of metabolic activity. In Mechanisms of Host Resistance to Infectious Agents, Tumors, and Allografts. R. M. Steinman, and R. J. North, editors. Rockefeller University Press, New York. 185-204.

24. Sananes, N., S. Weiller, E. E. Baulieu, and C. Le Goascogne. 1978. In vitro decidualization of rat endometrial cells. Endocrinology. 103:86-95.

25. Martin, G. R., and R. Timpl. 1987. Laminin and other basement membrane components. Ann. Rev. Cell Biol. 3:57-85.

26. Perri, R. T., G. Vercellotti, J. McCarthy, R. L. Vessella, and L. T. Furcht. 1985. Laminin selectively enhances monocyte-macrophage-mediated tumoricidal activity. J. Lab. Clin. Med. 105:30-35.

27. Shaw, L. M., and A. M. Mercurio. 1989. Interferon-gamma and lipopolysaccharide promote macrophage adherence to basement membrane laminin. J. Exp. Med. 169:303-308.

28. Giavazzi, R., L. Liotta, and I. Hart. 1982. Laminin inhibits the adhesion of a murine tumor of macrophage origin. Exp. Cell Res. 1140:315-322.

29. Gospodarawicz, D., R. Gonzalez, and D. K. Fujii. 1983. Are factors originating from serum, plasma, or cultured cells involved in the growth-promoting effect of the extracellular matrix produced by cultured bovine corneal endothelial cells? J. Cell. Physiol. 114:191202.

30. Moscatelli, D. 1988. Metabolism of receptor-bound and matrix-bound basic fibroblast growth factor by bovine capillary endothelial cells. J. Cell. Biol. 107:753-759.

31. Friedman, A., and D. I. Beller. 1988. The effect of adherence on the in vitro induction of cytocidal activity by macrophages. Immunology. 61:469-474.

32. Johnson, W. J., P. A. Marino, R. D. Schreiber, and D. O. Adams. 1983. Sequential activation of murine mononuclear phagocytes for tumor cytolysis: differential expression of markers by macrophages in the several stages of development. J. Immunol. 131:10381043.

33. Johnson, W. J., C. C. Whisnant, and D. O. Adams. 1981. The binding of BCG-activated macrophages to tumor targets stimulates secretion of cytolytic factor. J. Immunol. 127:1787-1803.

34. Tsunawaki, S., M. Sporn, A. Ding, and C. Nathan. 1988. Deactivation of macrophages by transforming growth factor-beta. $\mathrm{Na}$ ture (Lond.). 334:260-262.

35. Harrell, R. A., G. J. Cianciolo, T. D. Copelannd, S. Oroszlan, and R. Snyderman. 1988. Suppression of the respiratory burst of human monocytes by a synthetic peptide homologous to envelope proteins of human and animal retroviruses. J. Immunol. 136:35173520.

36. Tsunawaki, S., and C. F. Nathan. 1986. Macrophage deactivation: altered kinetic properties of superoxide-producing enzyme after exposure to tumor-cell conditioned medium. J. Exp. Med. 164:13191331.

37. Inaba, K., M. Kitaura, T. Kato, Y. Watanabe, and S. Muramatsu. 1986. Contrasting effect of alpha/beta and gamma-interferons on expression of macrophage Ia antigens. J. Exp. Med. 163:10301035.

38. Hunziker, R. D., and T. G. Wegmann. 1986. Placental immunorégulation. CRC Crit. Rev. Immunol. 6:245-285.

39. Lu, C. Y., R. W. Redline, C. M. Shea, L. B. Dustin, and D. B. McKay. 1989. Pregnancy as a natural model of allograft tolerance: interactions between adherent macrophages and trophoblast populations. Transplantation (Baltimore). 48:848-855.

40. Strom, T. B., J. W. Kupiec-Weglinski, and N. L. Tilney. 1985. On the mechanisms of rejection of vascularized organ allografts: review and an attempt at synthesis. In Progress in Transplantation. Volume 2. P. J. Morris and N. L. Tilney, editors. Churchill-Livingstone, New York. 126-146.

41. Head, J. R., B. L. Drake, and F. A. Zuckermann. 1987. Major histocompatibility antigens on trophoblast and their regulation: implications in the maternal-fetal relationship. Am. J. Reprod. Immunol. 15:12-18. 